\title{
Hidrógeno puro a partir de biogás: Efecto de la adición de agua en la etapa de reducción del proceso RSM+SIP con diferentes óxidos metálicos como transportadores de oxígeno
}

\author{
Beatriz López Barranco, Jaime Lachén, José Angel Peña, Javier Herguido \\ Catálisis, Separaciones Moleculares e Ingeniería de Reactores (CREG) \\ Instituto de Investigación en Ingeniería de Aragón (I3A) \\ Universidad Zaragoza, Mariano Esquillor s/n, 50018, Zaragoza, Spain. \\ Tel. +34-976762707, e-mail: jlachen@unizar.es
}

\begin{abstract}
Se estudia experimentalmente, en reactor de lecho fijo, el efecto de adicionar agua en la etapa de reducción del proceso combinado RSM+SIP para obtener hidrógeno puro a partir de biogás. Se comprueba la viabilidad para los óxidos empleados, evitándose además la acumulación de coque y la consiguiente necesidad de regeneración.
\end{abstract}

\section{Introducción}

Dada la actual problemática medioambiental, recobra importancia la denominada economía del hidrógeno obtenido de fuentes no fósiles [1]. En este trabajo se propone la producción de hidrógeno por el proceso "steam-iron" (SIP) [2], a partir de una fuente renovable como el biogás, frente al tradicional reformado de metano con vapor de agua. El método SIP permite la intensificación del proceso de producción y purificación de hidrógeno, en dos etapas. En la primera, un óxido metálico es reducido, para posteriormente en una segunda etapa, ser oxidado con vapor de agua produciéndose hidrógeno de alta pureza. Dada la naturaleza de los componenetes mayoritarios del biogás $\left(\mathrm{CH}_{4} \mathrm{y} \mathrm{CO}_{2}\right)$, la principal reacción en la etapa de reducción será el reformado seco de metano (RSM).

Un problema operacional es la posible formación de coque que provoca obturación del lecho y posible contaminación del hidrógeno con óxidos de carbono en la siguiente etapa de oxidación. Como solución se propone la adición de pequeños porcentajes de $\mathrm{H}_{2} \mathrm{O}$ que evitarían dicha acumulación, por gasificación de los depósitos carbonosos. Sin embargo dado su caracer oxidante, el $\mathrm{H}_{2} \mathrm{O}$ puede inhibir o ralentizar la primera etapa de reducción. En este trabajo se estudia el efecto producido por diferentes transportadores de oxígeno: ferrita de cobalto y óxido de hierro dopado (óxido 'triple'). Además, para mejorar la actividad del RSM, se usa simultáneamente catalizador de óxido de níquel [3].

\section{Experimental}

\section{Sólidos empleados}

Las ferritas de cobalto empleadas fueron aditivadas con alúmina produciendo una estequiometría $\mathrm{Al}_{0.53} \mathrm{Co} .8 \mathrm{Fe}_{1.6} \mathrm{O}_{4}$. Su síntesis se realizó mediante fusión conjunta [4]. El óxido 'triple' está compuesto por: $98 \%$ (p) $\mathrm{Fe}_{2} \mathrm{O}_{3}, 1,75 \%$ (p) $\mathrm{Al}_{2} \mathrm{O}_{3}$ y $0,25 \%$ (p) $\mathrm{CeO}_{2}$, sintetizándose según el método citratos [5]. El catalizador utilizado es un aluminato de níquel $\left(\mathrm{NiAl}_{2} \mathrm{O}_{4}\right)$ con un $10 \%(\mathrm{p})$ de $\mathrm{NiO}$ en exceso respecto al estequiométrico. Se obtuvo por el método de coprecipitación a pH creciente [6].

Todos los sólidos fueron molidos y tamizados hasta obtener un tamaño de partícula de 160-200 $\mu$ m.

\section{Reactor de lecho fijo}

En sistema experimental empleado consta de un reactor cilíndrico vertical de cuarzo, $\varnothing_{\mathrm{i}}=0,013 \mathrm{~m}$, con un lecho fijo de sólido de $2.5 \mathrm{~g}[75 \%$ (p) sólido activo, $25 \%$ (p) SiC como inerte]. El sólido activo era un $90 \%$ (p) óxido metálico (ferrita de cobalto o sólido triple) y $10 \%$ (p) catalizador.

Las etapas de reducción se han realizado a $700{ }^{\circ} \mathrm{C}$ con un caudal total de 250 (STP) $\mathrm{mL} / \mathrm{min}$ compuestos por: $25 \%$ (v) mezcla equimolar $\mathrm{CH}_{4} / \mathrm{CO}_{2}$ simulando un biogás desulfurado, $5 \%(\mathrm{v})$ $\mathrm{N}_{2}$ como patrón interno y diferentes proporciones de agua $(5-12,5 \%)$ y completando el balance con Ar. Las subsiguientes oxidaciones se realizaron con el mismo caudal total, pero compuesto de: $25 \%$ (v) $\mathrm{H}_{2} \mathrm{O}, 5 \%$ (v) $\mathrm{N}_{2}$, y resto $\mathrm{Ar}$, a una temperatura de $500{ }^{\circ} \mathrm{C}$. El seguimiento se realizó por cromatografía: $\mu$-GC Agilent 490 con columnas Molisieve $5 \AA$ y PoraPlot Q.

\section{Resultados y discusión}

Como ejemplo, se muestran los flujos molares de $\mathrm{H}_{2}$ y $\mathrm{CH}_{4}$ en la etapa de reducción alimentando 
diferentes proporciones de $\mathrm{H}_{2} \mathrm{O}$; tanto con ferrita de cobalto (figura 1) como con óxido 'triple' (figura 2).

En una primera etapa (A) se produce la reducción del sólido. En el caso de la ferrita, conjuntamente la del óxido de níquel del catalizador a níquel metálico, y la de la ferrita de cobalto a hierro y cobalto metálicos. Tanto Ni como Co catalizan la reacción de RSM produciendose $\mathrm{H}_{2}$ y $\mathrm{CO}$, (no mostrado), que son consumidos por el sólido en su reducción. Finalmente se alcanza una segunda etapa (B), cuyos flujos molares se corresponden con el equilibrio termodinámico, dominado por la reacción de Water-Gas-Shift. Para el óxido de hierro, la etapa A podría dividirse en dos subetapas: $A_{1}$ de rápida reducción del catalizador y de la hematita a magnetita y $\mathrm{A}_{2}$ de reducción de magnetita a hierro.

El efecto de la adición de agua para ambos sólidos, es similar. A mayores concentraciones de ésta, más se ralentiza la reducción del catalizador, provocando una mayor concentración de metano sin reaccionar al inicio de la reacción. Dada la formación de una atmosfera con menor poder reductor, el tiempo de reducción del sólido se ve incrementado. Sin embargo, la adición de agua supuso la no aparición de incrementos en la sobrepresión del sistema por acumulación de coque en el lecho, para ninguno de los dos sólidos. Las posteriores oxidaciones con vapor de agua (no mostradas) permitieron obtener un hidrógeno sin presencia de $\mathrm{CO}_{x}$, por lo que se garantiza la producción de hidrógeno de alta pureza.

\section{Conclusiones}

La adición de $\mathrm{H}_{2} \mathrm{O}$ en la etapa de reducción del proceso RSM+SIP con biogás, empleando como transportadores de óxigeno ferrita de cobalto u óxidos de hierro, supone cierta ralentización del

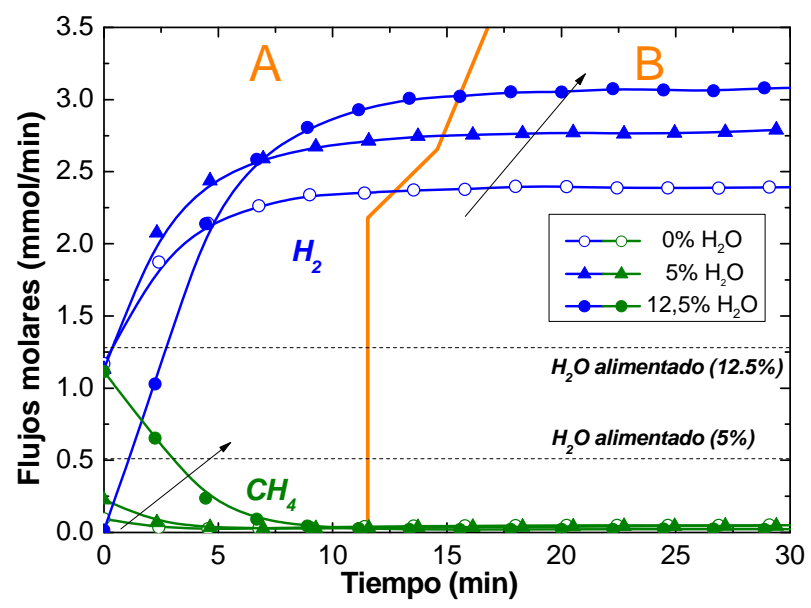

Figura 1: Primera reducción con lecho de ferrita de cobalto. Flujos de salida de $\mathrm{H}_{2}$ y $\mathrm{CH}_{4}$. proceso. Sin embargo elimina los problemas operacionales derivados de la acumulación de coque. Por ello, empleando bajas concentraciones de agua $(<5 \%)$ se consigue garantizar la operación continuada sin necesidad de intercalar una etapa de regeneración del lecho con aire. Además, se reduce el riesgo de contaminación por $\mathrm{CO}_{\mathrm{x}}$ del hidrógeno producido en las etapas de oxidación.

\section{REFERENCIA}

[1]. EHSAN. S, ABDUL. M. Hydrogen production from renewable and sustainable energy resources: Promising green energy carrier for clean development. Renewable and Sustainable Energy Reviews 57 (2016) 850-866.

[2]. MESSERCHMITT. A. Process of producing hydrogen. U.S. Patent 971,206 (1910).

[3]. PlOU J, DURÁN P., HERGUIDO J., PEÑA J.A. Hydrogen from synthetic biogas by catalyzed MDR and SIP: Screening of catalyst and iron oxide mixtures. Fuel 140 (2015) 470-476.

[4]. LÁZARO M.J., ECHEGOYEN Y., ALEGRE C., SUELVES I., MOLINER R., PALACIOS J.M. $\mathrm{TiO}_{2}$ as textural promoter on high loaded Ni catalysts for methane decomposition. International Journal of Hydrogen Energy 33 (13) (2008) 3320-3329.

[5]. KIRCHNEROVA J., ALIFANTI M., DELMON. Evidence of phase cooperation in the $\mathrm{LaCoO}_{3}-\mathrm{CeO}_{2}-$ $\mathrm{Co}_{3} \mathrm{O}_{4}$ catalytic system in relation to activity in methane combustion. Applied Catalysis A: General. 231 (1-2) (2002) 65-80.

[6]. AL-UBAID A., WOLF E.E. Steam reforming of methane reduced non-stoichiometric nickel aluminate catalysts. Applied Catalysis. 40 (1988) 73-85.

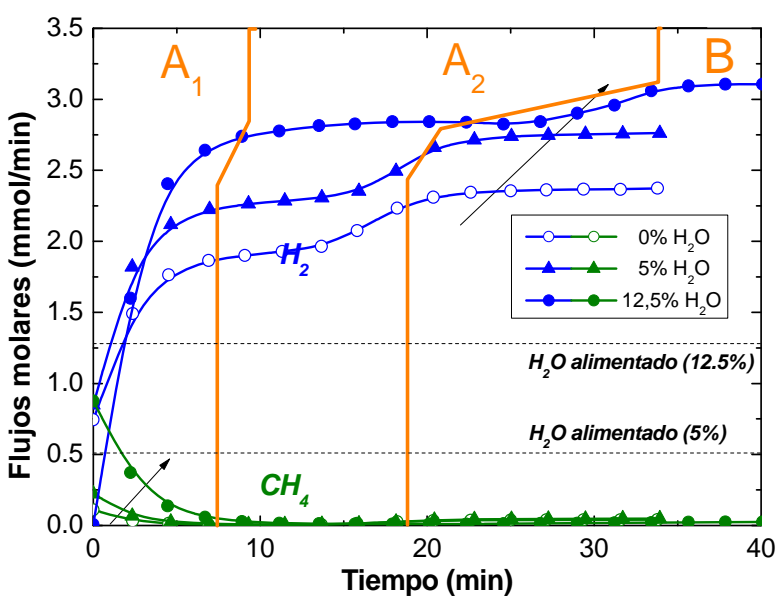

Figura 2: Primera reducción con lecho de óxido 'triple'. Flujos de salida de $\mathrm{H}_{2}$ y $\mathrm{CH}_{4}$. 